\title{
A simple kinetic model of preferential solvation in binary mixtures
}

\author{
Nikolai Kh. Petrov ${ }^{a}$, Alexander Wiessner ${ }^{b}$, Hubert Staerk ${ }^{b, *}$ \\ a Photochemistry Center, Russian Academy of Sciences, 117344 Moscow, Russia \\ ${ }^{\mathrm{b}}$ Max-Planck-Institut für biophysikalische Chemie, Abteilung Spektroskopie und Photochemische Kinetik, \\ Am Fassberg 11, D-37077 Göttingen, Germany
}

Received 8 August 2001; in final form 15 October 2001

\begin{abstract}
Based on experimental data a kinetic model is presented that adequately describes the main features of preferential solvation of a charge-transfer dipole in binary mixtures. 8-( $N, N$-dimethylamino)-11H-indeno[2,1-a]pyrene is used as fluorescent probe in toluene/DMSO mixtures. The observed saturation behavior can be understood in terms of microclusters of polar molecules surrounding the solute dipolar species. In such an approach, the size of the microclusters is mainly determined by interfacial surface tension. (c) 2001 Published by Elsevier Science B.V.
\end{abstract}

In recent years, scientific interest in preferential solvation in binary solvents, which has been motivated over decades by the challenge to establish a general mechanism for the influence of solvents on chemical reactions [1], seems revived after a fairly long time of neglect. The currently available methods of time-resolved optical spectroscopy, in particular the spectro-streak technique [2], allow the study of the dynamics of preferential solvation by monitoring the UV/Vis-spectral shift of an electronically-excited fluorescent probe. There are recent examples of such work dealing with nonspecific [3,4], and specific [5,6] interactions of probe molecules with a selective component of liquid binary solvent mixtures.

\footnotetext{
${ }^{*}$ Corresponding author. Fax: +49-551-2011501.

E-mail address: hstaerk@gwdg.de (H. Staerk).
}

Earlier steady-state (ss) fluorescence measurements [7] demonstrated that the dependence of the solvatochromic shift upon the volume fraction of a polar solvent component has a saturation behavior similar to that of a Langmuir adsorption isotherm. Based on this observation, Lippert put forward the hypothesis that the excited solute molecules have only a limited number (normally less than 10) of sites that can be occupied by polar solvent molecules. Recently it has been found [3] that preferential solvation also displays saturation behavior with respect to time. This is demonstrated with the fluorescent probe, 8-( $N, N$-dimethylamino)-11Hindeno[2,1-a]pyrene: Fig. 1, [Py(s)DMA], an intramolecular charge-transfer (CT) compound, whose fluorescence lifetime is about $23 \mathrm{~ns}$ and for which the characteristic time of solvation of the CT species is ca. 1 ns depending on the volume fraction of the polar solvent component. In 


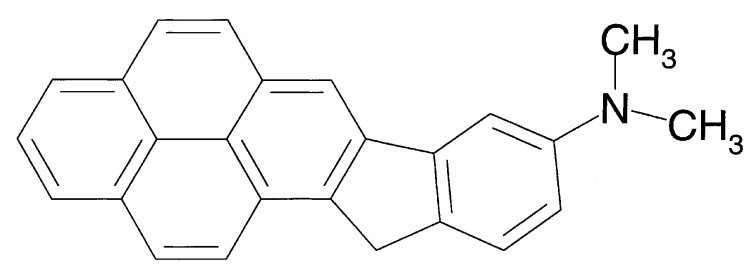

Fig. 1. Probe molecule Py(S)DMA.

mixtures of all compositions studied, the stationary fluorescence spectra coincide with those obtained by summation of the spectro-streak data collected over several nanoseconds after an excitation pulse. In other words, the process of solvation reaches a delayed saturation as time proceeds. Any known model dealing with the mechanism of creating a solvation shell seems unable to account clearly for this experimental fact.

The purpose of this communication is to present a simple kinetic model that adequately describes the main features of preferential solvation in binary mixtures. Our treatment is based mainly on the experimental data obtained in [3].

For the experimental description of preferential solvation, the so-called response function is usually used, defined as

$S(t)=\frac{\tilde{v}(t)-\tilde{v}(\infty)}{\tilde{v}(0)-\tilde{v}(\infty)}$,

where $\tilde{v}(t)$ is the position of the maximum of the probe fluorescence as a function of time. Considering the problem in terms of the Onsager model for the dipole interaction with a dielectric continuum, which was adapted to binary mixtures of components with considerably different dielectric properties (such as toluene/DMSO) [4], we have shown that (for small volume fractions of the polar component), the long-time tail of the response function is as follows:

$$
\begin{aligned}
S(t) & \approx \frac{1}{1+n(t)}, \quad \Delta \tilde{v}=(\tilde{v}(0)-\tilde{v}(\infty)) \\
& \approx \frac{9 m^{2}}{2 \varepsilon a^{3}},
\end{aligned}
$$

where $n(t)$ is the average number of polar molecules in the solvation shell of the fluorescent probe at time $t, \varepsilon$ is the dielectric permittivity of the polar component ( $\varepsilon=48.9$ for DMSO at $20{ }^{\circ} \mathrm{C}$ [Merck 2001]), $m$ is the dipole moment of the fluorescent probe, and $a$ is its radius.

Based on the collision theory of chemical reactions [8], the mechanism for creating the first solvation shell of polar molecules (e.g., DMSO) around electronically excited species B in binary mixtures can be written as a two-stage process (i.e., transport and solvation)

$\mathrm{A}+\mathrm{B} \underset{k_{-1}}{\stackrel{k_{1}}{\rightleftarrows}} \mathrm{AB} \stackrel{k_{2}}{\longrightarrow} \mathrm{B}$.

Here B is the excited (CT) dipole; A is the polar solvent molecule in the ground-state whose concentration is much larger than that of B and, therefore, essentially independent of time, i.e., $[\mathrm{A}] \approx[\mathrm{A}]_{0}$; and $\mathrm{AB}$ is the 'encounter pair' that is created when a polar molecule arrives in the neighborhood of a B molecule. Note that the fluorescence lifetime is supposed to be much longer than the characteristic time of solvation so that the fluorescent decay of B can be neglected during the solvation process. The latter, on the other hand, is much longer than the individual orientational relaxation time of the solvent components. It is also assumed that the components of the binary mixture differ strongly in terms of interaction with the solute molecule, $\mathrm{B}$, so that the non-polar component (e.g., toluene) is considered as an inert matrix.

The solvation stage is considered as a firstorder rearrangement of encounter pairs, i.e., jumping of molecules $A$ into the solvation shell of the $\mathrm{B}$ species. Being attached to $\mathrm{B}$, a solvated molecule A cannot diffuse anymore and the B species is ready to bind another polar molecule. It might be assumed that solvation is a catalytic reaction that creates a cluster of polar molecules A, with the electronically-excited dipole species as the 'catalyst'. However, solvation saturation might be taken into account formally by adding a process of poisoning of 'the catalyst' to the above scheme:

$\mathrm{B} \stackrel{k_{3}}{\rightarrow}$ the solvation-shell saturated species B

expecting that $\tau_{\mathrm{sol}}=\frac{1}{k_{3}} \sim 1 \mathrm{~ns}$.

The rate equations corresponding to schemes (3) and (4) can be written as follows: 


$$
\begin{aligned}
& \frac{\mathrm{d}[\mathrm{AB}]}{\mathrm{d} t}=k_{1}[\mathrm{~A}][\mathrm{B}]-\left(k_{-1}+k_{2}\right)[\mathrm{AB}], \\
& \frac{\mathrm{d}[\mathrm{B}]}{\mathrm{d} t}=-\frac{\mathrm{d}[\mathrm{AB}]}{\mathrm{d} t}-k_{3}[\mathrm{~B}],
\end{aligned}
$$

where brackets designate concentrations. Using the ss approximation, i.e., $\mathrm{d}[(\mathrm{AB})] / \mathrm{d} t=0$, we easily obtain

$$
[\mathrm{AB}]_{\mathrm{ss}}=\frac{k_{1}[\mathrm{~A}]_{0}[\mathrm{~B}]_{0} \exp \left(-k_{3} t\right)}{k_{2}+k_{-1}} .
$$

It is worth noting that the requirement $\left(k_{2}+k_{-1}\right) \approx k_{2} \gg k_{1}[\mathrm{~A}]_{0}$ is sufficient for the validity of the ss approximation and is fulfilled for all volume fractions of the polar component used [9].

Thus, the total number of solvated polar molecules is

$$
\begin{aligned}
N(t) & =\int_{0}^{t} k_{2}[(\mathrm{AB})] \mathrm{d} t^{\prime} \approx \int_{0}^{t} k_{2}[(\mathrm{AB})]_{\mathrm{ss}} \mathrm{d} t^{\prime} \\
& =\frac{k_{2} k_{1}[\mathrm{~A}]_{0}[\mathrm{~B}]_{0}}{k_{3}\left(k_{-1}+k_{2}\right)}\left(1-\exp \left(-k_{3} t\right)\right) .
\end{aligned}
$$

By definition, the average number of polar molecules solvated per excited dipole is $n(t)=$ $N(t) /[\mathrm{B}]_{0}$. So, from Eq. (7) we obtain
Table 1

Solvation numbers $n(\infty)$ vs volume fraction of DMSO as polar component admixed with toluene

\begin{tabular}{lllll}
\hline DMSO, vol\% & 3 & 4 & 6 & 7 \\
$n(\infty)$ & 1.5 & 2.1 & 7.0 & 8.0 \\
\hline
\end{tabular}

Other best-fit parameters are $\tilde{v}(\infty)=18221 \mathrm{~cm}^{-1}, \Delta \tilde{v}$ $=5384 \mathrm{~cm}^{-1}, \tau_{\mathrm{sol}}=1 / k_{3}=921 \mathrm{ps}$.

$n(t) \approx \frac{k_{1}[\mathrm{~A}]_{0}}{k_{3}}\left(1-\exp \left(-k_{3} t\right)\right)$,

where, for simplification, it is assumed that $k_{2} \gg k_{-1}$. This approximation seems reasonable at least for large volume fractions of the polar component. Evaluation of the dipole-dipole interaction of the solute $(m \sim 15-20 \mathrm{D})$ with the polar component (for example DMSO, $\mu \approx 4 \mathrm{D}$ ) confirms that it exceeds the energy of thermal motion at distances close to the radius of the first solvation shell [4].

From Eq. (8), it follows that for $t \rightarrow 0$, $n(t) \approx k_{1}[\mathrm{~A}]_{0} t$ (compare with the result obtained by a pure diffusion approach [4]) and in the limit of long times, i.e., for $t \rightarrow \infty, n(\infty) \approx k_{1}[\mathrm{~A}]_{0} / k_{3}$. Note that $\tau_{\text {diff }}=1 / k_{1}[\mathrm{~A}]_{0}$ is the characteristic time of diffusion transport of a polar molecule into the region immediately surrounding $\mathrm{B}$, whereas

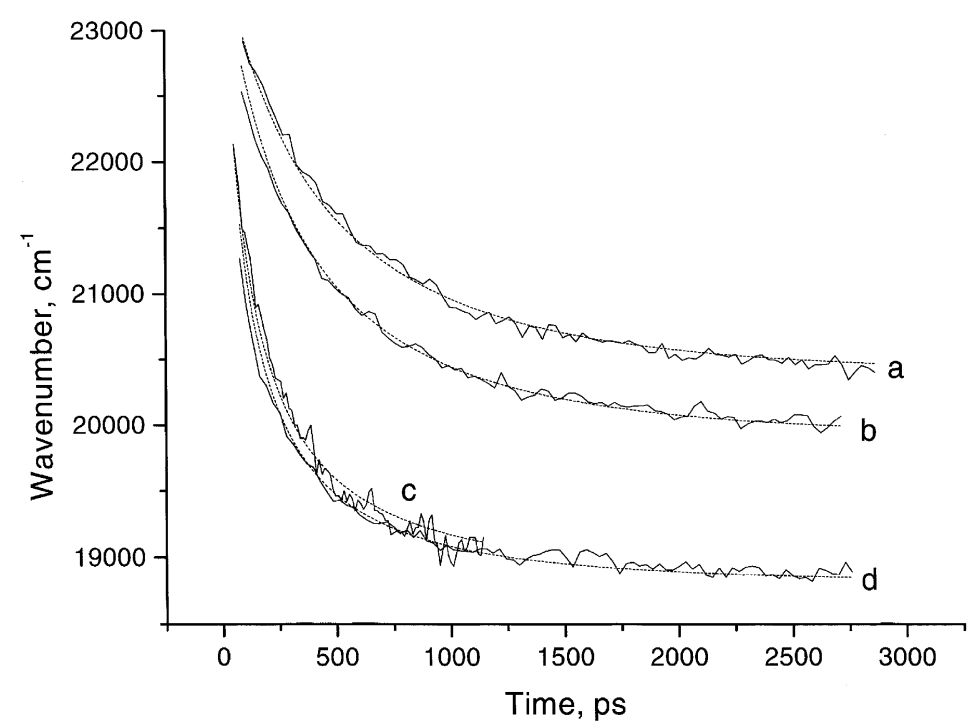

Fig. 2. Plot of transient solvatochromic shifts of the CT fluorescence band maximum vs time for Py(s)DMA in toluene/DMSO mixtures: (a) $3 \mathrm{vol} \%$ of DMSO (corresponding to $0.4 \mathrm{~mol}^{-1} \mathrm{l}^{-1}$ ); (b) $4 \mathrm{vol} \%$; (c) $6 \mathrm{vol} \%$; (d) $7 \mathrm{vol} \%$. The dotted lines are obtained with the best-fit parameters given in Table 1 . 
$\tau_{\mathrm{sol}}=1 / k_{3}$ is a characteristic time during which the excited dipole can absorb polar molecules into the solvation shell, so the latter result is physically understandable.

Insertion of $n(t)$ from Eq. (8) into Eq. (2) gives an expression that fits the experimental data reported in [3] surprisingly well (see Fig. 1). The best-fit parameters, presented in Table 1, are physically meaningful. The value obtained for $\Delta \tilde{v}=5384 \mathrm{~cm}^{-1}$ corresponds to $m \approx 15-20 \mathrm{D}$ and $a \approx 3 \AA$; and that for $\tilde{v}(\infty)=18221 \mathrm{~cm}^{-1}$ to ca. $11 \mathrm{vol} \%$ of DMSO. The characteristic solvation time $\tau_{\text {sol }}=1 / k_{3}=921 \mathrm{ps}$ is the same for all compositions of the mixtures and corresponds well to the experimental data. The values obtained for $n(\infty)$, which depend on the bulk concentration of polar molecules, are in agreement with the Lippert hypothesis [7]. However, if the establishment of equilibrium is determined by occupying the solute sites available for adsorption, then obviously the values of $n(\infty)$ should be equal for all solvent compositions while the characteristic time of solvation should vary. As mentioned above, the lifetime of the excited solute dipole ( $\geqslant 23 \mathrm{~ns})$ is long enough to adsorb decades of polar molecules because $\tau_{\text {diff }}=1 / k_{1}[A]_{0} \approx 260 \mathrm{ps}$, even for $3 \mathrm{vol} \%$ of DMSO (see Fig. 2). (The bimolecular diffusionlimited rate constant is determined by the viscosity of toluene and can be estimated as $k_{1}=1.13 \times 10^{10} \mathrm{~mol}^{-1} \mathrm{~s}^{-1}[10]$.) Therefore, the solvation number seems to be determined by the properties of the whole solution rather than only by the properties of the fluorescent solute. This can be understood by considering microclusters of polar molecules surrounding the solute dipolar species as nuclei of a new polar phase. In terms of such an approach, the size of the microcluster is mainly determined by surface tension of the in- terface, which might decrease (by analogy with the parachor - see for example [11]) as the bulk concentration of the polar solvent component increases. A decreasing surface tension results in an increase in the size of the microcluster, i.e. of $n(\infty)$, that is found by experiment.

\section{Acknowledgements}

The probe compound used in these investigations has been synthesized by Dr. W. Kühnle (MPI, Göttingen). N.Kh.P. thanks the Max Planck Society and the Russian Foundation for Basic Research (Grant no 01-03-32819) for support.

\section{References}

[1] C. Reichardt, Solvents and Solvent Effects in Organic Chemistry, second ed., Verlag Chemie, Weinheim, 1988; C. Reichardt, Chem. Rev. 94 (1994) 2319.

[2] A. Wiessner, H. Staerk, Rev. Sci. Instrum. 64 (1993) 3430.

[3] N.Kh. Petrov, A. Wiessner, T. Fiebig, H. Staerk, Chem. Phys. Lett. 241 (1995) 127.

[4] N.Kh. Petrov, A. Wiessner, H. Staerk, J. Chem. Phys. 108 (1998) 2326.

[5] F. Cichos, A. Willert, U. Rempel, C. von Borczyskowski, J. Phys. Chem. A 101 (1997) 8179.

[6] F. Cichos, R. Brown, U. Rempel, C. von Borczyskowski, J. Phys. Chem. A 103 (1999) 2506.

[7] E.L. Lippert, in: J.B. Birks (Ed.), Organic Molecular Photophysics, vol. 2, Wiley, New York, 1975, p. 1.

[8] A.M. North, The Collision Theory of Chemical Reaction in Liquids, Wiley, New York, 1964.

[9] L.P. Hammett, Physical Organic Chemistry: Reaction Rate, Equilibrium, and Mechanism, second ed., McGraw-Hill Book Company, New York, 1970.

[10] A.J. Gordon, R.A. Ford, The Chemist's Companion, Wiley, New York, 1972.

[11] O.R. Quayle, Chem. Rev. 53 (1953) 439. 